\title{
Una mirada al uso didáctico de las XO: percepciones y actitudes del profesorado de historia en Educación Secundaria
}

\section{Some considerations on the use of $X O$ as a teaching tool-perceptions and attitudes of high-school History teachers}

\begin{abstract}
María Guadalupe López Filardo
Master en Educación, Universidad ORT Uruguay. Licenciada en Ciencias Históricas, Universidad de la República. Profesora de Historia, Instituto de Profesores Artigas. Profesora de Historia en Educación Secundaria. Profesora de Teoría y Metodología de la Historia y de Historia de la Historiografía en el Instituto de Profesores Artigas.
\end{abstract}

Fecha de recibido: 20/01/2015

Fecha de aceptado: 31/03/2015

\section{Resumen}

La inclusión de tecnología digital en la enseñanza constituye actualmente un asunto de singular relevancia para la mejora educativa, a la vez que plantea enormes desafíos a las instituciones y particularmente a los docentes. Este fenómeno ha suscitado una serie de interrogantes vinculadas a las percepciones que tienen los profesores y las actitudes que adoptan respecto a la integración de tecnología en sus prácticas de enseñanza y a los factores que inciden en dicho proceso.

El presente artículo contiene las líneas centrales de una investigación destinada a penetrar en el sentido y los significados que construye el profesorado de Historia de Educación Secundaria en torno a la introducción en el aula de las computadoras portátiles (XO) del Plan Ceibal, los criterios pedagógicos que impulsan su uso y sus implicancias tanto para la enseñanza de esta disciplina como para la resignificación del rol docente.

Enmarcado en una metodología cualitativa de enfoque etnográfico, se realizó un estudio de casos de tipo múltiple, contando con la colaboración de diez profesoras de historia que desempeñan funciones en liceos públicos de Montevideo. Mediante un modelo interpretativo de características fenomenológica y hermenéutica, se procuró describir y comprender los casos objeto de estudio a través de entrevistas en profundidad, observación de clases y análisis documental.

La evidencia empírica recogida mostró una significativa heterogeneidad de situaciones en cuanto a los modos de apropiación e integración de esta tecnología y la aparición de algunos cambios incrementales en el rol docente. Se observó, asimismo, cómo los enfoques didácticos atribuidos al uso de las XO permanecen aún sujetos a modelos tradicionales de gestión curricular, lo que estaría indicando la necesidad de repensar la formación de nuevas competencias para el trabajo docente y la creación de condiciones que faciliten y estimulen la propagación de prácticas pedagógicas transformadoras.

Palabras clave: computadoras portátiles Plan CEIBAL, educación secundaria, profesorado de Historia, percepciones y actitudes, prácticas de enseñanza. 


\begin{abstract}
The inclusion of digital technology in teaching and training processes is particularly relevant to their improvement and, at the same time, challenges both educational institutions and teachers. Many questions have arisen in this sense since the implementation of the new tool. How do teachers perceive the use of technology? How can they integrate technology to their usual teaching practices? Which are the factors intervening in this process?

This paper contains the main lines of a research aiming at penetrating the sense and the meanings associated by high-school History teachers with the use of Ceibal Project's XO laptops in class work. We also take into consideration the pedagogical criteria supporting the use of XO and its implications on both History teaching methods and the role of History teachers.

A multiple-case study has been conducted applying an ethnographically-focused qualitative methodology. Ten female History teachers working in state high-schools in Montevideo have willingly participated in this study. A phenomenological hermeneutical interpretation model -including in-depth interviews, class observation and document analysis- has been developed for the purpose of learning, describing and properly understanding the cases in question.

The empirical evidence collected has shown remarkably heterogeneous situations concerning the modes of acquisition and integration of the new technology by teachers, as well as the intervention of certain changes in the scope of the teachers' role. Likewise, the didactical approaches stemming from the use of XO laptops still adhere to traditional curriculum-management patterns. Consequently, it seems necessary to develop new teaching skills, as well as suitable conditions that facilitate and encourage the generalisation of innovative pedagogic practices.
\end{abstract}

Key words: Plan Ceibal laptops (XO), high-school education, History teachers, perceptions and attitudes, teaching practices.

\title{
Introducción
}

El vertiginoso desarrollo experimentado por las Tecnologías de la Información y la Comunicación (TIC) ha contribuido a transformar los espacios educativos en procura de integrar a las personas a un mundo complejo y globalizado que exige conocimientos y habilidades renovables permanentemente. En este nuevo escenario, y alineado con las actuales políticas de inclusión socioeducativa que se vienen impulsando en toda la región, Uruguay lanzó en el año 2006 el programa “Conectividad Educativa de Informática Básica para el Aprendizaje en Línea" (Plan Ceibal)1. Concebido en el marco de una iniciativa gubernamental claramente identificada con la inclusión social y la democratización para el desarrollo de oportunidades en la sociedad de la información, el programa se sustenta “...en el principio de igualdad de oportunidades, que pretende disminuir la brecha digital y potenciar el uso de las tecnologías para generar aprendizajes de calidad en los alumnos" (Báez y Pérez, 2008:25).

La investigación producida a nivel nacional sobre los impactos tecnológicos y sociales del Plan Ceibal revela que las acciones iniciales, destinadas a asegurar las condiciones de acceso de niños, jóvenes y familias de sectores populares a las computadoras portátiles (denominadas XO), han merecido una valoración social altamente positiva, lo que constituye un objetivo deseable y de indiscutible efecto democratizador. No obstante, "el objetivo social asociado a cualquier tecnología educativa (el lápiz, el libro de texto y el portátil) no es el éxito de la mencionada tecnología sino la mejora del proceso y el entorno en el que tienen lugar la enseñanza y el aprendizaje" (OCDE, 2010:13). En consecuencia, uno de los focos centrales del programa está orientado a los usos de la tecnología en las prácticas 
educativas. “Las tecnologías digitales impulsadas a través de la implementación del Plan Ceibal en Uruguay potencian modelos pedagógicos que además de habilitar cambios en las prácticas de enseñanza, [...] interpelan fuertemente a todos los actores de la educación, generando la necesidad imperiosa de una reformulación del acto educativo" (Báez y García, 2011:106).

El proceso de inclusión de las XO en el sistema educativo uruguayo -que al presente cubre la totalidad de escuelas primarias y centros públicos de educación media básica2 del país- muestra que pese a los esfuerzos realizados durante la etapa de consolidación del Plan, todavía se están intentando dilucidar las dificultades que entrañan, para los docentes, la integración pedagógica de esta tecnología y sus efectos en la transformación de las prácticas de enseñanza. En el contexto de esta problemática se consideró relevante desarrollar una investigación que contribuyera a avanzar en la descripción y en una mejor comprensión del modo en que los profesores de enseñanza secundaria perciben la nueva realidad impuesta por la generalización de las XO en el aula, sus expectativas y la manera en que actúan frente a esta innovación, procurando a su vez identificar los factores que intervienen en dicho proceso.

\section{Planteo general de la investigación}

La incorporación de tecnologías digitales en la enseñanza es una experiencia común a cualquier disciplina, pero no cabe duda de que el conocimiento de la naturaleza epistemológica del campo disciplinar resulta fundamental para analizar y comprender sus prácticas. Por esta razón, el área de la presente investigación se circunscribió únicamente a profesores de historia, en primer lugar por tratarse del ámbito de especialización de la investigadora y, en segundo lugar, por las especiales características de esta disciplina cuya didáctica ha experimentado en las últimas décadas notables cambios conceptuales y metodológicos que obedecen, en parte a su evolución paradigmática y, en parte, a la fuerte influencia de las TIC, lo que ha contribuido a renovar la visión de los docentes respecto de las finalidades de su enseñanza y sus opciones metodológicas.

El interés por el tema estuvo ligado, por ende, a una preocupación por ampliar la comprensión acerca de la presencia de las TIC en la enseñanza de la Historia y los modos en que es concebida y puesta en práctica por los docentes. Ello dio lugar al planteo de un objetivo central de investigación: conocer y comprender cómo es percibido, por los profesores de historia, el proceso de inclusión de la tecnología digital impulsado por el Plan Ceibal en el primer ciclo de Enseñanza Secundaria, sus formas de apropiación y de integración a la enseñanza.

A partir de dicho objetivo central se formularon cuatro objetivos específicos que habrían de guiar el estudio:

- Identificar, definir y categorizar las percepciones de los profesores de historia sobre la inclusión y el uso de las XO en el Ciclo Básico de Secundaria.

- Analizar e interpretar los factores que inciden en las actitudes y en la mayor o menor predisposición de los profesores de historia a incorporar las XO en la enseñanza.

- Describir los diferentes modos en que los profesores de historia se apropian de esta tecnología y la integran a sus prácticas cotidianas de aula.

- Identificar y caracterizar las percepciones de los profesores de historia acerca de su propio rol en el nuevo contexto generado por el Plan Ceibal. 


\section{Antecedentes y fundamentación teórica}

Como actividad intencional estrechamente vinculada a las creencias, formación y experiencia de los docentes, la enseñanza remite irremediablemente a las teorías y paradigmas que les sirven de sustento y que se reflejan explícita o implícitamente en sus prácticas. "Las teorías comprenden concepciones acerca de cómo se enseña y cómo se aprende, así como los principios que sustentan las decisiones sobre variadas cuestiones tales como [...] las funciones que como docentes deben cumplir y las misiones que la escuela tiene como resultado de su compromiso con la sociedad y con cada individuo" (Camilloni, 2010:43).

Para comprender lo que sucede durante el proceso de enseñanza es necesario conocer las teorías que subyacen al pensamiento de los profesores y que determinan sus formas de actuación. Son precisamente esas teorías las que, junto a otros factores, determinan los estilos pedagógicos que adoptan los profesores en sus clases.

Estudios relativos a las concepciones docentes sobre las finalidades de la enseñanza de la Historia (Pozo, Asensio y Carretero, 1989; González Muñoz, 2002; Prats, 2007; Benejam, 2008), comparten la idea de que es preciso indagar los significados socialmente construidos por los profesores acerca de la enseñanza para hallar el grado de coherencia entre la acción y el pensamiento. Según afirman, a pesar de que aún persisten estilos de enseñanza de tipo transmisivo, en la actualidad el empleo de estrategias didácticas por descubrimiento y de metodologías basadas en la investigación ha cobrado amplia difusión entre el profesorado de historia. Pilar Maestro González (1993), comentando los cambios que viene experimentando la enseñanza de la disciplina en el nivel medio destaca, entre otros aspectos, el abandono paulatino de la organización frontal y unidireccional del aula (adecuada a la clase magistral) y el papel de "expositor" del docente, quien progresivamente se va convirtiendo en "preparador" de un plan y un material a utilizar en clase, adaptándose al trabajo en pequeños grupos, favoreciendo la consulta de distintas fuentes y estimulando la investigación.

De acuerdo con lo que señala esta autora, la Historia ha dejado de ser "recibida" para pasar a ser "descubierta" o "investigada" y luego "construida" (Maestro González, 1993:143-155). Este imbricado camino de renovación metodológica que desafía la labor de los profesores de historia se ha visto interpelado, en los últimos tiempos, por la irrupción de lo que Area Moreira (2009) Ilama "nuevas tecnologías digitales", que además de generar cambios en la organización y presentación del conocimiento promueven la reformulación del rol docente. Al tiempo que el profesor deja de ser la principal fuente de información para sus estudiantes, se ve impelido a adoptar un nuevo perfil que le exige conocer el material disponible, saber seleccionarlo, ofrecer información en una diversidad de formas para responder a las necesidades de los alumnos y a las exigencias curriculares, y actuar como mediador en los procesos de aprendizaje. Según sostienen Barberà, Mauri y Onrubia (2010), en la sociedad de la información el profesor cumple, entre otras funciones, las de “...proveedor de recursos, facilitador del aprendizaje, supervisor académico, guía para sus alumnos, colaborador del grupo-clase, motivador del saber, consumidor de información, activador de conocimientos previos, planificador escrupuloso, asesor de técnicas de estudio y estrategias de aprendizaje, evaluador continuo, gestor de conocimientos, potenciador de autoaprendizaje" (Barberà, Mauri y Onrubia, 2010:22).

No obstante, la redefinición del rol docente no depende exclusivamente de la incorporación de tecnologías sino de las capacidades, actitudes y creencias pedagógicas de los profesores y, en buena medida, de que su uso sea compatible con sus teorías previas. En opinión de Jesús Valverde (2011), el profesorado adopta una innovación cuando considera que la 
misma ha de aportarle alguna ventaja con respecto a la práctica docente a la que sustituye, y el éxito depende de la innovación pedagógica que pueda desarrollar mediante su empleo. Afirma, en consecuencia, que si los profesores se hallan suficientemente motivados para usar tecnología en sus aulas, lo han de hacer a pesar de los obstáculos que se les presenten.

Según los resultados de una encuesta elaborada por el Departamento de Monitoreo y Evaluación del Plan Ceibal (2011) respecto a la inclusión de tecnologías digitales en la Educación Media, su uso está determinado por los potenciales beneficios que el profesorado les atribuye, de acuerdo con los objetivos perseguidos. Cuando las XO son consideradas únicamente como herramientas de búsqueda, acceso y procesamiento de la información (lo que sin duda constituye una necesidad insoslayable en la sociedad actual) se las valora positivamente, pero su aplicación a los procesos de enseñanza y de aprendizaje merece apreciaciones menos favorables. Incluso -como advierte Michael Fullan (2013) en un informe relativo a los avances de las dos primeras fases de implementación del Planes posible "que los docentes permitan o incluso alienten a los alumnos a usar la XO, sin que esto repercuta en cómo preparan las clases, y sin vínculos claros con los objetivos curriculares" (Fullan et al., 2013:16). Tales consideraciones coinciden con resultados de investigaciones internacionales, en las que se sostiene que la simple incorporación de tecnología no asegura procesos de mejora o de innovación de la enseñanza. Según Magdalena Claro (2010), una implementación exitosa de prácticas innovadoras depende no sólo de las características de la innovación sino también de los factores implicados en ella. La clave, por consiguiente, se halla en el uso que se hace de la tecnología y “...el impacto que produzca -ya sea bueno o malo- depende en gran medida de los contextos en los que se usa, las motivaciones de quienes la usan y el propósito con que intentan usarla" (Buckingham, 2008:103).

Una investigación reciente sobre los aspectos pedagógicos del Plan Ceibal revela que existen tres dimensiones cuyo estudio debiera profundizarse: la relación entre el tipo de uso de la tecnología y las prácticas de enseñanza en cada asignatura, las condiciones institucionales y pedagógicas en que se usan las XO, y el papel que juegan las características sociales e individuales de los docentes en su apropiación y forma de uso de esta tecnología (Vaillant, 2013:48). De tal forma, la perspectiva pedagógica y el rol docente son primordiales para garantizar una adecuada articulación de la tecnología con la propuesta pedagógica en cuyo marco se desarrolla, como parte de un proceso de innovación.

\section{Diseño metodológico}

El enfoque de una investigación cualitativa -centrado en el estudio de realidades subjetivas e intersubjetivas como objetos legítimos de conocimiento- proporciona, por su naturaleza fenomenológica, un marco comprensivo que ayuda a analizar en profundidad la complejidad de determinadas situaciones. Partiendo de esta idea, se optó por una lógica investigativa anclada en el paradigma cualitativo desde el que pudieran inferirse algunas claves interpretativas que facilitaran la construcción de significados. "La investigación cualitativa es ilimitadamente creativa e interpretativa. El investigador no se limita a dejar atrás el campo con una pila de material empírico y la voluntad de poner por escrito sus descubrimientos. Las interpretaciones cualitativas surgen de un proceso de construcción" (Denzin y Lincoln, 2012:90).

Se diseñó un modelo indagativo y analítico cimentado en un estudio de casos de tipo múltiple (Stake, 2010), empleando una muestra intencional en la que la selección de las unidades de análisis estuvo sujeta a criterios teóricos definidos a priori. Se trabajó con profesores titulados en Historia, con cargo efectivo en Educación Secundaria, una actuación docente mínima de ocho años y experiencia en el trabajo con las XO, y cuyas 
trayectorias profesionales estuvieran validadas por miembros referentes de la comunidad académica. A efectos de facilitar el acceso al campo (en función del tiempo disponible para desarrollar la investigación) y contar con una buena acogida, se dispuso que los profesores seleccionados estuvieran desempeñando funciones en liceos de Montevideo y que manifestaran voluntariamente su disposición a participar en el estudio. De esta manera, mediante un muestreo en cadena o "bola de nieve" (Tójar Hurtado, 2006:188), se fue conformando una muestra cuya composición estructural contempló tres tipos de variables: socioculturales, espaciales y temporales.

Para la recolección de datos se seleccionaron instrumentos basados en técnicas directas (entrevistas en profundidad, semiestructuradas y observación no participante de clases) e indirectas (análisis documental). El empleo combinado de ambas técnicas obedeció no sólo a la intención de enriquecer la información a través de la visión particular de cada una sino de lograr que su interrelación y complementariedad contribuyeran a una mejor comprensión de los datos.

Como puede observarse en el siguiente esquema, el diseño adoptado respondió a un modelo "interactivo" (Maxwell, 1996) en el que el uso de conectores y líneas entre los componentes -representando uniones de influencia o de implicación en ambos sentidosindica un proceso dinámico y recurrente en el que las supuestas fases fueron, en realidad, las acciones que permitieron ir profundizando en el proceso de investigación. Al decir de Maxwell (1996:2), “...se trata de una estructura interconectada y flexible”, que a la vez de identificar los componentes clave del diseño, subraya las relaciones entre éstos y presenta una estrategia para crear relaciones coherentes y manejables entre los mismos. 


\section{GRÁFICO 1. DISEÑO GENERAL DE LA INVESTIGACIÓN}

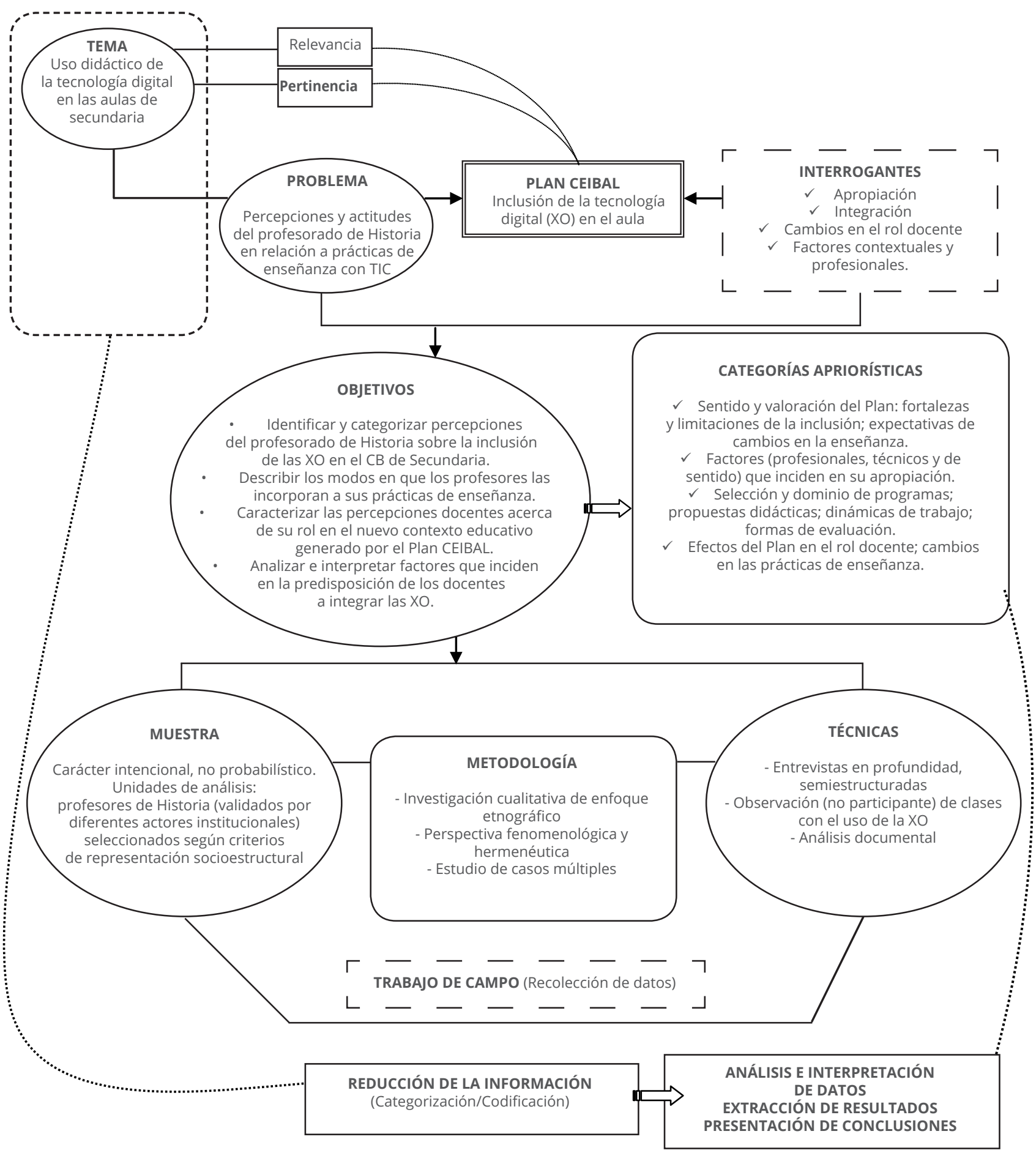

Fuente: elaboración propia 


\section{Tratamiento y análisis de los datos}

La investigación -desarrollada a lo largo del año 2013- abarcó un total de diez casos, considerándose cada docente un caso en sí mismo. "Lo que interesa en la muestra cualitativa, que opera con un número reducido de casos, es la profundidad del conocimiento del objeto de estudio y no la extensión de la cantidad de unidades" (Mejía Navarrete 2011:166).

El perfil de la muestra, integrada por diez docentes del género femenino, respondió a las siguientes características:

\section{CUADRO N 1. CARACTERÍSTICAS QUE DEFINEN LA SINGULARIDAD DE LOS CASOS}

\begin{tabular}{|c|c|c|c|c|c|c|c|c|c|}
\hline \multirow[b]{2}{*}{$\begin{array}{l}\text { Edad } \\
\text { (entre) }\end{array}$} & \multirow[b]{2}{*}{$\begin{array}{c}\text { Cantidad } \\
\text { de } \\
\text { Profesores }\end{array}$} & \multirow[b]{2}{*}{$\begin{array}{l}\text { Titulación } \\
\text { de Grado }\end{array}$} & \multirow[b]{2}{*}{$\begin{array}{l}\text { Cantidad } \\
\text { de } \\
\text { Profesores }\end{array}$} & \multirow[b]{2}{*}{$\begin{array}{l}\text { Posgrados y/o } \\
\text { especialización }\end{array}$} & \multirow[b]{2}{*}{$\begin{array}{l}\text { Cantidad } \\
\text { de } \\
\text { Profesores }\end{array}$} & \multirow{2}{*}{$\begin{array}{l}\text { Antigüedad } \\
\text { docente } \\
\text { (Grado en el } \\
\text { Escalafón) }\end{array}$} & \multirow[b]{2}{*}{$\begin{array}{l}\text { Cantidad } \\
\text { de } \\
\text { Profesores }\end{array}$} & \multirow{2}{*}{$\begin{array}{l}\text { Nivel de } \\
\text { formación } \\
\text { en TIC }\end{array}$} & \multirow[b]{2}{*}{$\begin{array}{c}\text { Cantidad } \\
\text { de } \\
\text { Profesores }\end{array}$} \\
\hline & & & & & & & & & \\
\hline \multirow{2}{*}{$\begin{array}{l}31 \text { y } 40 \\
\text { años }\end{array}$} & \multirow{2}{*}{3} & \multirow{2}{*}{$\begin{array}{l}\text { Profesor de } \\
\text { Historia }\end{array}$} & \multirow{2}{*}{10} & \multirow{4}{*}{$\begin{array}{l}\text { Maestría en Didác- } \\
\text { tica de la Historia }\end{array}$} & \multirow{2}{*}{$\begin{array}{c}1 \\
\text { (completa) }\end{array}$} & $2^{\circ}$ & 2 & \multirow{2}{*}{ Básico } & \multirow{2}{*}{4} \\
\hline & & & & & & $3^{\circ}$ & 1 & & \\
\hline \multirow{2}{*}{$\begin{array}{l}41 \text { y } 50 \\
\text { años }\end{array}$} & \multirow{2}{*}{4} & \multirow{2}{*}{$\begin{array}{l}\text { Licenciado en } \\
\text { Historia }\end{array}$} & \multirow{2}{*}{1} & & \multirow{2}{*}{$\begin{array}{c}4 \\
\text { (en curso) }\end{array}$} & $4^{\circ}$ & 1 & \multirow{2}{*}{ Avanzado } & \multirow{2}{*}{5} \\
\hline & & & & & & $5^{\circ}$ & 1 & & \\
\hline \multirow{2}{*}{$\begin{array}{l}51 \text { y } 62 \\
\text { años }\end{array}$} & \multirow{2}{*}{3} & \multirow{2}{*}{$\begin{array}{l}\text { Licenciado en } \\
\text { Psicología }\end{array}$} & \multirow{2}{*}{1} & \multirow{2}{*}{$\begin{array}{l}\text { Tecnicatura en Infor- } \\
\text { mática }\end{array}$} & \multirow{2}{*}{1} & $6^{\circ}$ & 2 & \multirow{2}{*}{ Experto } & \multirow{2}{*}{1} \\
\hline & & & & & & $7^{\circ}$ & 3 & & \\
\hline
\end{tabular}

El estudio de las entrevistas se fue desenvolviendo mediante un análisis fenomenológico y a la vez de producción de conocimiento, adoptando en tal sentido la metáfora "del entrevistador como viajero" que propone Kvale (2011:43-44), según la cual entrevista y análisis constituyen fases entrelazadas de construcción de conocimiento. Se buscó identificar y delimitar, a través de los discursos, un conjunto de categorías descriptivas (o macro-categorías) que reflejasen las percepciones y actitudes del profesorado de historia con relación a cuatro grandes dimensiones o bloques temáticos asociados a los objetivos específicos formulados en el marco general de la investigación. Para ello, previo al análisis de los datos y con base en lo sugerido por Cisterna (2005:65), se definió un conjunto de "categorías apriorísticas" que, alineadas con las preguntas y objetivos de la investigación, se fueron desglosando en respectivas subcategorías de análisis. 


\section{GRÁFICO II. DIAGRAMA DE LAS CATEGORÍAS Y SUBCATEGORÍAS APRIORÍSTICAS}

A partir de una "matriz parcialmente ordenada" (Miles \& Huberman, 1994:278) se fue

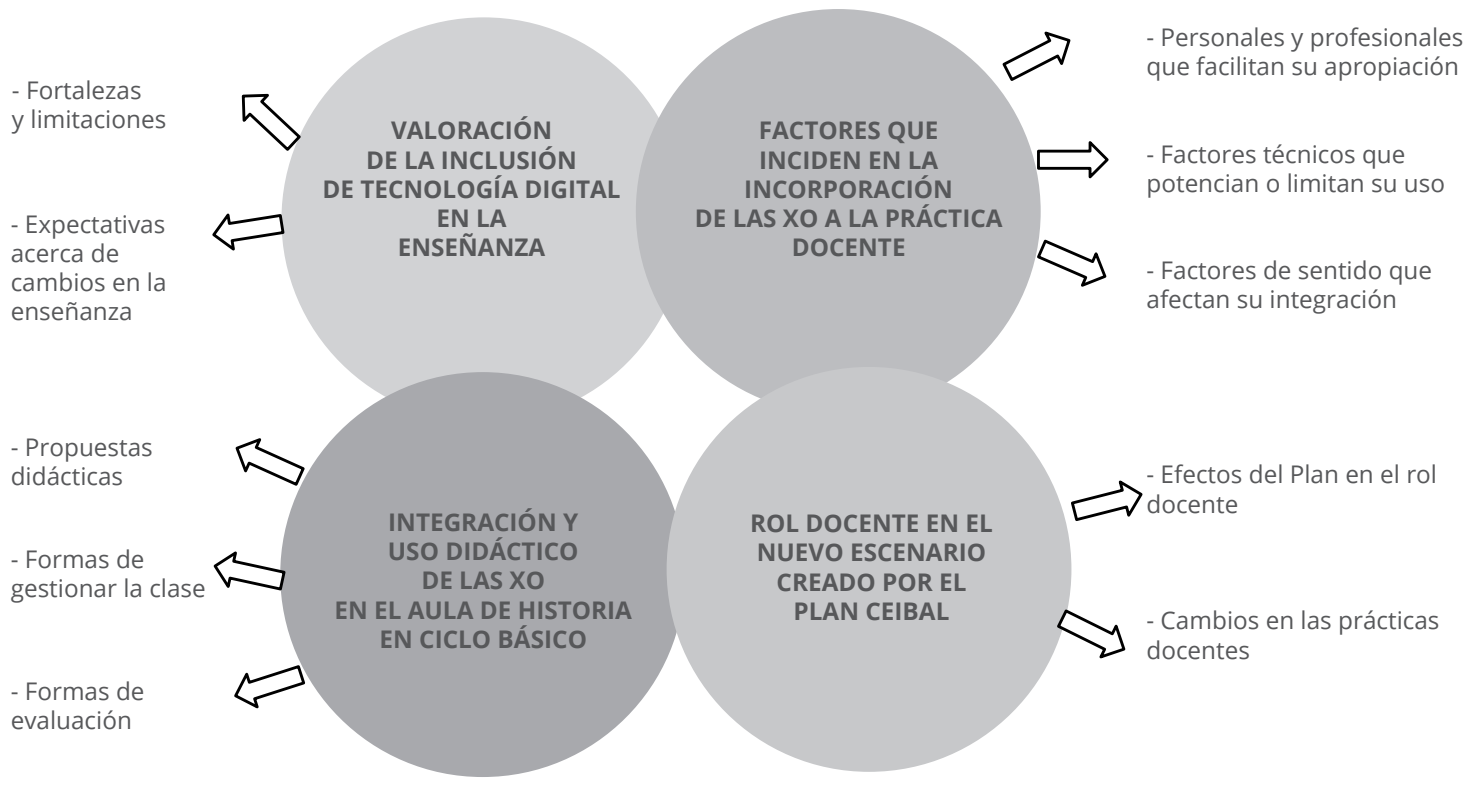

reuniendo la información obtenida en las diez entrevistas, procediéndose luego al análisis y codificación correspondientes a cada caso y estableciéndose relaciones entre las citas y los códigos de manera de llegar a presentaciones descriptivas y ordenadas conceptualmente. Un procedimiento similar se llevó a cabo con las observaciones de clases y los documentos, de modo que tras la identificación, ordenamiento y clasificación de los datos se emprendió la construcción de conceptos teóricos y explicativos de la interpretación, tratando -como indican Coffey y Atkinson (2005:183)- de trascender, es decir, de "ir más allá" de los datos mediante una explicación cuidadosamente construida, con el fin de comprender los procesos genéricos entre un caso y otro.

La sistematización en el tratamiento y análisis de los datos se fue dando en forma espiralada (como se ilustra en el Gráfico III), puesto que la reducción, el análisis descriptivo y la interpretación se influyen mutuamente, al tiempo que son procesos paralelos. Vale decir que, acabada cada etapa, se retornó con frecuencia a la fase anterior para reiniciarla con información más precisa. Este proceso supuso la lectura reiterada del material, "...con la intención de reconstruir la realidad en su contexto concreto y, además, con la intención de reflexionar acerca de la situación vivida para comprender lo que ocurre" (Mejía Navarrete, 2011:48). 


\section{GRÁFICO III. REPRESENTACIÓN DEL PROCESO DE TRATAMIENTO Y ANÁLISIS DE LOS DATOS}

\section{NIVELES}

3. Interpretación y extracción de conclusiones (Construcción del marco interpretativo marco interpretativo y la estructura final)

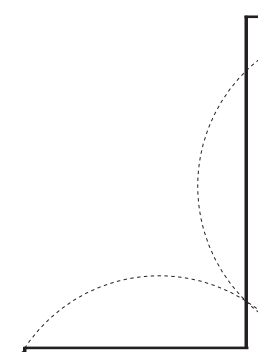

Desarrollo y testeado de proposiciones para construir el marco de análisis

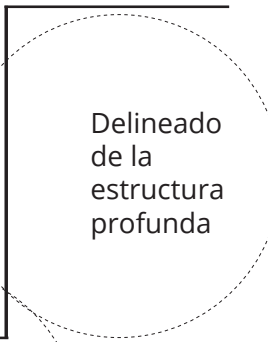

Síntesis e integración de datos para redacción del informe y principales conclusiones

Triangulación hermenéutica Convergencia de datos Segundo nivel de análisis: identificación de frecuencias de aparición de códigos Redacción de hallazgos por categorías

2.

Disposición/ transformación de los datos

(Reempaquetamiento y agregación de datos)
Búsqueda de relaciones e identificación de tendencias en los datos en general
Primer resumen descriptivo

\section{1.}

Reducción de la información

(Resumen y empaquetamiento de datos)
Codificación de entrevistas, observaciones y documentos (proceso deductivo/inductivo)

Segmentación y selección de las unidades de significado Escritura de notas analíticas en relación con el marco conceptual
Desgrabación y sinopsis de las entrevistas individuales

Primera revisión de observaciones y documentos y creación de textos

\section{categorías} de codificación

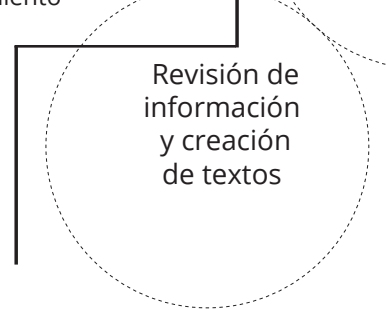

Fuente: elaboración propia en base a la adaptación de un gráfico creado por Miles \& Huberman (1994:92)

Para asegurar la validez y confiabilidad de los datos se optó por una "triangulación hermenéutica", es decir, la "acción de reunión y cruce dialéctico de toda la información pertinente al objeto de estudio surgida en una investigación por medio de los instrumentos correspondientes, y que en esencia constituye el corpus de resultados de la investigación" (Cisterna, 2005:69). Se triangularon datos procedentes de distintas fuentes lo que permitió, como "método solapado" (Rodríguez Sabiote et al., 2005:150) facilitar la comprensión y compensar posibles deficiencias. Se realizó primeramente una triangulación de primer orden (intracasos) a efectos de contrastar la información aportada por cada profesor en la entrevista con lo observado directamente en la clase y lo apreciado en los documentos, para desde allí generar nuevos procesos interpretativos. "El paradigma cualitativo constituye un intercambio dinámico entre la teoría, los conceptos y los datos con retroinformación 
y modificaciones constantes de la teoría y de los conceptos, basándose en los datos obtenidos" (Cook y Reichardt, 1995:66). Luego se realizó una triangulación de segundo orden (intercasos) a fin de determinar posibles resultados similares. El empleo de este procedimiento de triangulación en dos niveles se apoya en una doble lógica: la obtención de una visión múltiple y enriquecida durante la recolección de los datos, desde distintas fuentes y con diferentes técnicas, y el control de calidad en la interpretación al posibilitar lecturas que pueden contrastarse empíricamente con otra serie similar de datos.

\section{Resultados}

Sistematizados los datos recogidos en la fase empírica, la evidencia obtenida permitió establecer relaciones entre: i) las percepciones de las profesoras sobre el proceso de inclusión de las XO; ii) los factores que influyen en sus actitudes para apropiarse e incorporar esta tecnología al trabajo de aula; iii) los cambios percibidos en cuanto a su rol en este nuevo escenario, y; iv) los modos en que se integra el uso de las XO a las prácticas de enseñanza de la historia.

\section{Valoración general de la tecnología digital implementada por el Plan Ceibal}

Si bien la política de inclusión digital de los adolescentes que cursan la educación media básica cuenta con una valoración positiva por parte del profesorado, desde el punto de vista de la mejora de los procesos de enseñanza y de aprendizaje su sentido es aún poco comprendido. Se visualiza a la XO como una herramienta que facilita el acceso a la información e incrementa el contenido didáctico de las propuestas docentes, pero en líneas generales podría decirse que todavía no se ha logrado percibirla como una oportunidad para repensar y renovar las prácticas de enseñanza. Este fenómeno obedece a un conjunto interrelacionado de factores, sustentados principalmente en el cambio de ideas y prácticas pedagógicas que lo acompañan.

De acuerdo con estimaciones de las profesoras integrantes de la muestra, uno de los obstáculos parte de la forma en que se implementó el Plan Ceibal en Secundaria. La aparente ausencia de una planificación que atendiera de manera prioritaria el enfoque pedagógico, a la vez que generó malestar entre el profesorado no alentó la creación de sentido que debiera acompañar toda innovación. El sentido se construye desde las subjetividades y, por tanto, más allá de las resistencias que pueda suscitar el cambio tecnológico (debido fundamentalmente a las modificaciones que impone en las rutinas habituales del trabajo docente) existen modos diferentes de percibirlo y de ponderar sus beneficios. En este caso, las resistencias han hecho prevalecer las demandas más que las actitudes reflexivas sobre los efectos del Plan.

\section{Condiciones que afectan la apropiación y el uso de la tecnología}

La mayor o menor predisposición de los docentes a apropiarse e integrar tecnología en sus propuestas de enseñanza se basa en ciertas representaciones más o menos generalizadas del valor educativo atribuido a las XO, a las que tiende a considerarse como elemento mediador en los procesos de enseñanza y recurso facilitador de aprendizajes. Estas visiones imprimen un sentido diverso al uso de la herramienta, como puede apreciarse en el siguiente cuadro, ilustrado con algunos testimonios de las profesoras. 


\section{CUADRO Nº 2. RAZONES MÁS FRECUENTES QUE DETERMINAN EL USO DE LAS XO}

\begin{tabular}{|l|l|}
\hline $\begin{array}{l}\text { Motivación y satisfacción } \\
\text { de intereses de estudiantes }\end{array}$ & $\begin{array}{l}\text { “...creo que al usar la XO los chicos se sienten un poco más interesados en } \\
\text { los temas y las actividades que se les proponen y más involucrados en el } \\
\text { proceso de aprendizaje”. (P6) }\end{array}$ \\
\hline $\begin{array}{l}\text { Facilidades de uso y acceso } \\
\text { a información }\end{array}$ & $\begin{array}{l}\text { “...entiendo que hay que aprovechar la predisposición y la alfabetización } \\
\text { que los estudiantes ya tienen en este tipo de tecnología, para poder } \\
\text { desarrollar habilidades que son transversales a cualquier disciplina (....... } \\
\text { yo empecé a usar la XO como forma de facilitar a los estudiantes el } \\
\text { acceso a elementos que tradicionalmente incorporaba en mis clases de } \\
\text { otra manera (...) para indicarles algunas páginas Web en las que pueden } \\
\text { consultar o de las que pueden bajar información..." (P2) }\end{array}$ \\
\hline $\begin{array}{l}\text { Ampliación del tiempo } \\
\text { de clase y desarrollo de } \\
\text { habilidades comunicativas }\end{array}$ & $\begin{array}{l}\text { “...creo con el poco tiempo de clase que tenemos, hay que pensar en que } \\
\text { esta tecnología te permite crear lo que algunos llaman un 'aula expandida', } \\
\text { o sea, que todo no se agota en el tiempo de la clase ...los alumnos se } \\
\text { mantienen en contacto conmigo o entre ellos mismos, o con otros } \\
\text { compañeros, y están vinculados a una comunidad virtual”. (P5) }\end{array}$ \\
\hline $\begin{array}{l}\text { Modificaciones en las formas } \\
\text { de participación estudiantil }\end{array}$ & $\begin{array}{l}\text { “...aspiro a que mis alumnos adquieran más autonomía en sus procesos } \\
\text { de aprender y considero que usando esta herramienta pueden encontrar } \\
\text { una forma de relacionarse más activamente con la información”. (P3) } \\
\text { “...al trabajar con la XO los alumnos sienten que tienen un papel más } \\
\text { protagónico, porque pueden integrar ellos mismos recursos multimedia...." } \\
\text { (P10) }\end{array}$ \\
\hline
\end{tabular}

Pese al reconocimiento explícito de los beneficios que promueve el uso de las XO, existe sin embargo una combinación de factores de distinta naturaleza que influyen en las actitudes de los docentes dificultando el logro de los propósitos educativos. Las limitaciones más significativas son de orden pedagógico e institucional. Entre las primeras se percibe, en primer término, insuficiente formación para el empleo de tecnologías informáticas. Al parecer, la política de formación desarrollada hasta el momento por el Plan Ceibal no ha satisfecho las expectativas del profesorado que expresa no alcanzar buen dominio de las competencias necesarias para trabajar con las XO y menos aún para desarrollar una innovación que trascienda las paredes del aula. Asociado a este factor, otro de singular importancia es el referido al "tiempo pedagógico", aspecto recurrente en casi todas las dimensiones de la investigación y de muy compleja resolución. El profesorado reclama tiempo para explorar las posibilidades que ofrece la herramienta, aprender a utilizarla con eficacia y adquirir destrezas en el manejo de los recursos; tiempo para seleccionar y preparar contenidos digitales, planificar propuestas didácticas y especialmente para desarrollarlas en el aula; tiempo para reunirse con colegas, compartir ideas, dilemas y dificultades que se plantean en la práctica en contextos determinados.

Concomitantemente, entre los factores de orden institucional se percibe: escasa flexibilidad de los centros educativos para habilitar tiempos y espacios que propicien el intercambio profesional; insuficiente prestación de apoyos pedagógicos y tecnológicos calificados que orienten el proceso de integración tecnológica y provean estrategias de acompañamiento al profesorado. Si bien se advierte entusiasmo en algunos docentes que, motivados por el uso de la tecnología dedican tiempo y esfuerzos a capacitarse y tratar de impulsar modificaciones en sus prácticas, la inercia institucional tiende al mantenimiento de rutinas que no facilitan los cambios. Incluso los pequeños avances incrementales que perciben las profesoras en cuanto a la apropiación y el uso de la tecnología no se ven potenciados debido a la ausencia de una política interna de difusión del conocimiento y de la experiencia profesional. Como señala Carlos Marcelo García, "El aislamiento de los profesores está favorecido evidentemente por la arquitectura escolar, que organiza las escuelas en módulos independientes, así como por la distribución del tiempo y el espacio y la existencia de normas de autonomía y privacidad entre los profesores" (Marcelo, 2002:12). 


\section{Cambios en el rol docente}

En el orden práctico se distinguen diversas formas de aproximación al uso de las XO, lo que significa que su frecuentación no es ajena a la cotidianeidad del aula de Historia. En las prácticas observadas se reconocen propuestas variadas, aún cuando la mayoría responde a un tipo de uso preferentemente "instrumental", lo que implica que pese a la incorporación de nuevos recursos destinados a crear entornos virtuales más propicios para la generación de aprendizajes persiste la aplicación de prácticas más bien tradicionales, con baja presencia de elementos favorecedores de creatividad y autonomía en el estudiantado. Como puede observarse en el siguiente cuadro, existen algunas convergencias en los diez casos estudiados. Todas las profesoras integran en alguna medida esta herramienta a sus propuestas de enseñanza, y -ya fuere en forma individual o colaborativa- todas han participado en la producción y el diseño de materiales didácticos en soporte electrónico a partir de herramientas de la Web 2.0. La mayoría dispone de un blog como apoyo a los contenidos curriculares, y asegura contar con suficientes referencias para orientar a sus estudiantes en la búsqueda de información disponible en Internet.

\section{CUADRO N 3. CARACTERIZACIÓN DE LOS MODOS EN QUE SE UTILIZAN LAS XO}

\begin{tabular}{|c|c|c|c|c|}
\hline \multirow{3}{*}{ Frecuencia y oportunidad de uso } & \multicolumn{2}{|c|}{ Se la utiliza en forma } & \multicolumn{2}{|c|}{ Ocasional más que habitual } \\
\hline & \multirow{2}{*}{\multicolumn{2}{|c|}{$\begin{array}{l}\text { Se la emplea en } \\
\text { actividades }\end{array}$}} & \multicolumn{2}{|c|}{$\begin{array}{l}\text { Preferentemente domiciliarias } \\
\text { (con menor frecuencia } \\
\text { presenciales) }\end{array}$} \\
\hline & & & \multicolumn{2}{|c|}{ Individuales y grupales } \\
\hline $\begin{array}{l}\text { Empleo del software disponible en } \\
\text { la XO }\end{array}$ & \multicolumn{2}{|c|}{$\begin{array}{l}\text { En forma casi exclusiva } \\
\text { (ocasionalmente) }\end{array}$} & \multicolumn{2}{|c|}{$\begin{array}{l}\text { Compartido con otros programas } \\
\text { (mayormente) }\end{array}$} \\
\hline $\begin{array}{l}\text { Modalidades de uso por parte de los } \\
\text { estudiantes }\end{array}$ & $\begin{array}{l}\text { Acceso a } \\
\text { información } \\
\text { contenida } \\
\text { en la red }\end{array}$ & $\begin{array}{l}\text { Exposición } \\
\text { de } \\
\text { contenidos }\end{array}$ & $\begin{array}{l}\text { Ejercicios de } \\
\text { aplicación y/o } \\
\text { reforzamiento } \\
\text { de la } \\
\text { información }\end{array}$ & $\begin{array}{l}\text { Actividades que } \\
\text { propician la } \\
\text { comprensión y } \\
\text { autorregulación } \\
\text { del aprendizaje }\end{array}$ \\
\hline $\begin{array}{l}\text { Fuentes de información utilizadas en } \\
\text { la planificación docente y/o sugeridas } \\
\text { a los estudiantes }\end{array}$ & $\begin{array}{l}\text { Sitios Web } \\
\text { específicos }\end{array}$ & $\begin{array}{l}\text { Portales } \\
\text { educativos }\end{array}$ & $\begin{array}{l}\text { Revistas } \\
\text { digitales }\end{array}$ & $\begin{array}{l}\text { Productos } \\
\text { digitales } \\
\text { preelaborados }\end{array}$ \\
\hline $\begin{array}{l}\text { Producción y desarrollo de materiales } \\
\text { didáctico-digitales por los docentes }\end{array}$ & Blog & Wiki & Web Quest & $\begin{array}{l}\text { Presentaciones } \\
\text { (PPT, Prezi, etc.) }\end{array}$ \\
\hline
\end{tabular}

En cuanto a las posibilidades que brinda la integración de tecnologías digitales para la actualización de los enfoques didácticos y metodológicos de la disciplina enseñada, pudo advertirse que en la mayor parte de los casos examinados la misma no ha sido explotada en todas sus potencialidades. Aun cuando se observan prácticas que potencian el trabajo colaborativo, estimulan el empleo de dinámicas de enseñanza más reflexivas y procuran brindar mayor protagonismo y autonomía a los estudiantes, aquéllas parecieran ser insuficientes para que los alumnos utilicen la tecnología en la producción de conocimientos y en un aprovechamiento eficaz de la información.

En lo que refiere a la evaluación, algunas profesoras toman en cuenta su uso para calificar a sus estudiantes pero sin incursionar en nuevas estrategias de evaluación. 


\section{Profesores de Historia y prácticas de enseñanza con XO}

Los resultados del estudio refuerzan la idea concebida en el marco teórico de la investigación de que las percepciones docentes, entendidas como representaciones sociales explicitas, obedecen a los campos disciplinares de formación y a los contextos específicos de actuación.

En un esfuerzo por asociar dichas percepciones a los usos didácticos de las XO fue posible establecer que las mismas están fuertemente arraigadas en la disciplina impartida y en factores culturales que se hallan en la base de cualquier intento por modificar las formas de actuación pedagógica. Las subcategorías emergentes de este análisis están focalizadas en las dimensiones prioritarias, o al menos más recurrentes en los discursos y la acción de cada docente. Quienes comparten percepciones similares en cuanto a la enseñanza de la Historia centran sus prácticas en determinados saberes y experiencias, no sólo didácticos sino también disciplinares y, consecuentemente, atribuyen un papel diferente a la integración de la tecnología.

\section{CUADRO N 4. CARACTERIZACIÓN DE LAS PERCEPCIONES Y ACTITUDES DEL PROFESORADO DE HISTORIA EN RELACIÓN A LAS FORMAS DE INTEGRACIÓN DE LAS XO}

\begin{tabular}{|c|c|c|c|}
\hline $\begin{array}{l}\text { ENSEÑANZA DE LA } \\
\text { HISTORIA COMO: }\end{array}$ & Conocimiento del pasado & $\begin{array}{l}\text { Desarrollo del } \\
\text { pensamiento histórico }\end{array}$ & $\begin{array}{l}\text { Construcción del } \\
\text { contexto social }\end{array}$ \\
\hline ÉNFASIS EN: & Contenidos disciplinares & $\begin{array}{l}\text { Saberes pedagógicos y } \\
\text { disciplinares }\end{array}$ & $\begin{array}{l}\text { Investigación y } \\
\text { experimentación }\end{array}$ \\
\hline $\begin{array}{l}\text { MODOS DE USO } \\
\text { FRECUENTE DE XO }\end{array}$ & $\begin{array}{l}\text { Exposición de contenidos } \\
\text { Búsqueda de información } \\
\text { Ejercitación y afianzamiento }\end{array}$ & $\begin{array}{l}\text { Interacción didáctica y } \\
\text { social } \\
\text { Aplicación y } \\
\text { transferencia de } \\
\text { aprendizajes }\end{array}$ & $\begin{array}{l}\text { Trabajo en entornos } \\
\text { virtuales } \\
\text { Uso de distintas fuentes } \\
\text { Transformación } \\
\text { de información en } \\
\text { conocimiento }\end{array}$ \\
\hline
\end{tabular}

Las profesoras que ponen el acento en el conocimiento de los hechos históricos según son presentados por las principales corrientes historiográficas, privilegian las explicaciones sobre sus causas y efectos. "Para mí enseñar historia es ayudar a los alumnos a entender cómo han vivido los hombres en el pasado, ayudarlos a que puedan tomar conciencia de los actos humanos y puedan comprender los problemas que los hombres han tenido que enfrentar en el transcurso de los siglos y las respuestas que han dado a esos problemas" (P4). Consideran que los recursos tecnológicos que incorporan están destinados preferentemente a apoyar la exposición de los contenidos, mientras que el uso de las XO por parte de los estudiantes está orientado principalmente a la búsqueda de información y la ejercitación, es decir que se las utiliza como elemento reforzador de los procesos de enseñanza y aprendizaje.

Quienes opinan que el objetivo fundamental de enseñar historia es ayudar a los estudiantes a pensar históricamente entienden que los contenidos deben contribuir al desarrollo de un pensamiento crítico, la construcción de una identidad colectiva o la formación ciudadana. Vale decir que el contenido relevante de la Historia es de orden transversal y estratégico. “... la enseñanza de la historia es una herramienta indispensable para que los adolescentes y los jóvenes puedan pensar críticamente y encontrar explicaciones a la realidad actual" (P8). 
“...la misión que tenemos los profesores que enseñamos Historia es ayudar a formar a los jóvenes como ciudadanos del mundo" (P9).

Las prácticas de estas profesoras, centradas en saberes pedagógicos y disciplinares, buscan motivar a los estudiantes mediante el uso transformador de la tecnología. Por lo general no usan las XO para exponer contenidos sino que intentan modelar las posibilidades que ofrece esta herramienta como medio de interacción didáctica y social procurando, a través de trabajos colaborativos, que los estudiantes establezcan una relación más fluida con los contenidos y que transfieran sus aprendizajes a otros contextos.

Las profesoras que perciben la enseñanza de la Historia como construcción del contexto social enfatizan las relaciones entre su concepción epistemológica y la práctica de su enseñanza: lo importante es que los estudiantes realicen actividades que les permitan integrar conceptos generales, discernir, relacionar y valorar los procesos históricos desde distintos ámbitos sociales, políticos y económicos. "La Historia tiene que ser entendida como un saber en permanente construcción [...] Esa es para mí la base del conocimiento histórico; no es el descubrimiento de una verdad sino el descubrimiento de la relación existente entre dos hechos a la luz de un sistema de valoraciones. [...] no se trata de hacer que los alumnos descubran los conocimientos históricos como lo haría un historiador sino de organizarles tareas que los familiaricen con la aplicación de un pensamiento que cuestiona, que establece contradicciones, que busca soluciones, que interroga, que diseña posibles estrategias..." (P6).

Aprovechando la familiaridad de los estudiantes con las $\mathrm{XO}$, estas profesoras crean entornos de aprendizaje en los que se incentiva el acceso a la información para su transformación en conocimiento a través del diseño de actividades en las que la tecnología se integra naturalmente a las propuestas de trabajo. Planifican sus clases en base a aportes seleccionados de distintas fuentes (portales educativos, revistas digitales, sitios Web específicos) y sus secuencias didácticas se orientan a generar diálogo entre y con los estudiantes.

En suma, desde la perspectiva de una transformación de las prácticas de enseñanza de una disciplina como la Historia, es posible afirmar que los modos de integración y uso de las XO responden básicamente a dos aspectos: las percepciones de los docentes sobre las finalidades de su enseñanza y los enfoques epistemológicos y didácticos que definen sus opciones metodológicas.

\section{Conclusiones}

Los hallazgos surgidos del presente estudio aportan evidencias que hacen visibles algunos aspectos clave que podrían ampliar la mirada sobre el uso didáctico de las XO.

Un elemento vital en cualquier proceso de transformación educativa es la adhesión consciente y voluntaria de quienes deben llevarlo adelante, por lo que su viabilidad depende, en buena medida, de las motivaciones y los significados que los profesores otorgan a sus prácticas. Al igual que cualquier otro tipo de innovación en educación, el proceso de inclusión de las XO es dinámico y requiere de la construcción de una cultura de colaboración, que en un ambiente de creatividad y compromiso con la tarea estimule la propagación de prácticas pedagógicas transformadoras. Esto conlleva, por una parte, la necesidad de repensar el trabajo con la tecnología desde una perspectiva más abierta y flexible -especialmente en cuanto a la organización y gestión del sistema- de manera de ofrecer a los docentes oportunidades de adquirir competencias tecnológicas generales y aplicables a la didáctica de la disciplina o área en que se desenvuelven a partir de sus necesidades concretas y a través de una formación horizontal que incite a la colaboración profesional. Sería necesario, por consiguiente, continuar desarrollando estrategias 
destinadas a fortalecer la calidad de la formación inicial y estimular la formación continua de los docentes en servicio, concediendo especial atención a la integración de la tecnología desde su relación con la práctica docente.

Otra de las cuestiones controversiales gira en torno al rol docente y es la relativa a la reproducción de las prácticas educativas. A pesar de la importancia y el valor que el profesorado otorga a las XO, no hay evidencias de que el cambio se haya concretado en culturas o prácticas innovadoras. En los modelos didácticos empleados ordinariamente continúan conviviendo formas de trabajo previas a la inclusión de esta tecnología y que tienden a modelar el uso de la nueva herramienta, lo que podría estar indicando que hasta el momento su presencia no habría contribuido a transformar las formas de enseñar. Ello se debe, posiblemente, a que las estructuras de pensamiento de los docentes se mantienen en gran medida ancladas en paradigmas tradicionales, de modo que una innovación como la que promueve el uso de las XO, en lugar de modificar las prácticas termina siendo absorbida por modelos que dan continuidad a las lógicas de enseñanza dominantes. En consecuencia, para generar cambios en las percepciones y actitudes del profesorado sobre la enseñanza en este nuevo escenario de tecnologías digitales, es necesario fortalecer el sentido pedagógico de los cambios.

Finalmente, en relación a los usos didácticos de la XO, no cabe duda de que habría que continuar trabajando los modos de integración al trabajo del aula, hasta que esta tecnología -como señala Cabero (2007)- se haga invisible a los ojos de docentes y estudiantes. Para ello es importante contar con estudios que profundicen en el análisis de las prácticas educativas, ligado esto a una cierta concepción de la profesionalidad y de la búsqueda de la mejora. A diferencia del análisis llevado a cabo por cualquier disciplina académica, el análisis de la práctica de enseñar utilizando las XO no tiene que ver con la producción de conocimientos (en el sentido de tratar de saber o de entender algo que se ignora) sino con una reorientación de las líneas de trabajo y particularmente del rol docente. Dicho proceso debiera ser fruto de una construcción colectiva que se asemeje -empleando una metáfora inspirada en un texto de DuFour y Burnette- al cultivo de un jardín. “Un jardín no es lineal, algunos elementos mueren cuando otros están naciendo. Un jardín está influido por factores externos e internos. Sus factores más vitales se sitúan en el subsuelo y no se pueden ver fácilmente. Sobre todo un jardín es frágil y difícil de mantener. Incluso el más florido se cubrirá de maleza si no se cuida" (DuFour y Burnette, 2002:27).

\section{Referencias bibliográficas}

Area Moreira, M. (2009). Introducción a la Tecnología Educativa. Accedido el 8 de julio, 2013, desde https://campusvirtual.ull.es/ocw/file.php/4/ebookte.pdf

Baez, M. \& García, J. M. (2011). Desafíos a la pedagogía en la era digital. En Baez, M. et al. El modelo CEIBAL. Nuevas tendencias para el aprendizaje (pp. 98-116). Montevideo: ANEP/CEIBAL.

Baez, M. \& Pérez Burger, M. (2008). CEIBAL en la escuela. Quehacer Educativo, Año XVIII (91), 25-30.

Barberá, E., Mauri, T. \& Onrubia, J. (2010). Cómo valorar la calidad de la enseñanza basada en las TIC. Pautas e instrumentos de análisis. Barcelona: Graó.

Benejam, P. (2008). Las finalidades de la educación social. En Benekam, P. \& Pagés, J. (coords.) Enseñar y aprender Ciencias Sociales, Geografía e Historia en la Educación Secundaria (pp. 33-51). Barcelona: Horsori. 
Buckingham, D. (2008). Más allá de la tecnología. Aprendizaje infantil en la era de la cultura digital. Buenos Aires: Manantial.

Cabero, J. (2007). Las necesidades de las TIC en el ámbito educativo: oportunidades, riesgos y necesidades. Tecnología y Comunicación Educativas, Año 21 (45), 4-19.

Camilloni, A. R. W. (2010). Los profesores y el saber didáctico. Buenos Aires: Paidós.

Claro, M. (2010). La incorporación de tecnologías digitales en educación. Modelos de identificación de buenas prácticas. CEPAL. Accedido el 4 de marzo, 2013, desde http:// www.cepal.org/es/publicaciones/la-incorporacion-de-tecnologias-digitales-en-educacionmodelos-de-identificacion-de

Cisterna Cabrera, F. (2005). Categorización y triangulación como procesos de validación del conocimiento en investigación cualitativa. Theoria, 14 (1): 61-71. Accedido el 22 de julio, 2013, desde http://www.redalyc.org/articulo.oa?id=29900107

Coffey, A. \& Atkinson, P. (2005). Encontrar el sentido a los datos cualitativos. Estrategias complementarias de investigación. Medellín: Universidad de Antioquia.

Cook, T. D. \& Reichardt, Ch. S. (1995). Métodos cualitativos y cuantitativos en investigación evaluativa. ( $2^{a}$ ed.) Madrid: Morata.

Denzin, N. K. \& Lincoln, I. S. (coords.) (2012). El campo de la investigación cualitativa. Manual de investigación cualitativa. (Vol. I). Barcelona: Gedisa.

Dufour, R. \& Burnette, B. (2002). Pull out negativity by its roots. Journal of Staff Development, 23 (3) 27-30. Accedido el 14 de marzo, 2014, desde http://prodev.sdale.org/Facilitator resources_TIA_UBD/Articles/Article_Pull_out_negativity by its_roots[1].doc

Fullan, M., Watson, N. \& Anderson, S. (2013). CEIBAL: Los próximos pasos. Informe final. Accedido el 3 de febrero, 2013, desde http://www.Ceibal.org.uy/docs/FULLAN-Versionfinal-traduccion-Informe-Ceibal.pdf

González Muñoz, M. C. (2002). La enseñanza de la historia en el nivel medio. Situación, tendencias e innovaciones. Madrid: Anaya.

Kvale, S. (2011). Las entrevistas en Investigación Cualitativa. Madrid: Morata.

Maestro González, P. (1993). Epistemología histórica y enseñanza. Ayer, (12), 135-181.

Marcelo García, C. (2002). La profesión docente en momentos de cambios. ¿Qué nos dicen los estudios internacionales? Estudios e investigaciones. CEE Participación Educativa, marzo 2011, 49-68. Accedido el 24 de marzo, 2014, desde http://www.mecd.gob.es/revista-cee/ pdf/n16-marcelo-garcia.pdf

Maxwell, J. A. (1996). Un modelo para el diseño de investigaciones cualitativas. Qualitative research design. An Interactive Approach. Applied Social Research methods (41) California, EEUU: Sage Publications. Traducción María Luisa Graffigna. 1-13. Accedido el 6 de agosto, 2013, desde http://www.catedras.fsoc.uba.ar/guemure/bibliografia/Maxwell1.pdf 
Mejía Navarrete, J. (2011). Problemas centrales del análisis de datos cualitativos. Revista Latinoamericana de Metodología de la Investigación Social, (1) 1. Argentina. 47-60 Accedido el 7 de agosto, 2013, desde http://www.relmis.com.ar/ojs/index.php/ relmis/article/view/11/1

Miles, M. B. \& Huberman, A. M. (1994). Qualitative Data Analysis. An Expanded Sourcebook, $\left(2^{\text {nd }}\right.$. ed.). Thousand Oaks, California: Sage Publications.

OCDE (2010). 1:1 en Educación. Prácticas actuales, evidencias del estudio comparativo internacional e implicaciones en políticas. Accedido el 17 de enero, 2013, desde http:// recursostic.educacion.es/blogs/europa/media/blogs/europa/informes/1a1_en_educacion OCDE.pdf

Plan Ceibal (2011). Encuesta a docentes de Educación Media pública sobre acceso, dominio y uso de herramientas TIC. Departamento de Monitoreo y Evaluación del Plan Ceibal.

Pozo, J. I., Asensio, M. \& Carretero, M. (1989). Modelos de aprendizaje-enseñanza de la Historia. En Carretero, M., Pozo, J. I. \& Asensio, M. La Enseñanza de las Ciencias Sociales (pp. 211-240). Madrid: Visor.

Prats, J. (2007). Dificultades para la enseñanza de la Historia en la Educación Secundaria: reflexiones ante la situación española. En Lima, L., Salazar, J., Bonilla, F. \& Acevedo, M. (comp.) Historia I. Antología (pp. 75-78). México: Secretaría de Educación Pública.

Rodríguez Sabiote, C., Lorenzo Quiles, O. \& Herrera Torres, L. (2005). Teoría y práctica del análisis de datos cualitativos. Proceso general y criterios de calidad. Revista Internacional de Ciencias Sociales y Humanidades, vol. (XV), 133-154. Accedido el 18 de setiembre, 2013, desde http://www.redalyc.org/pdf/654/65415209.pdf

Stake, R. (2010). Investigación con Estudio de Casos. Madrid: Morata.

Tójar Hurtado, J. C. (2006). Investigación Cualitativa. Comprendery actuar. Madrid: La Muralla.

Vaillant, D. (2013). Las políticas TIC en los sistemas educativos de América Latina. Caso Uruguay. Buenos Aires: UNICEF. Accedido el 22 de octubre, 2013, desde http://www.unicef.org/ argentina/spanish/Uruguay_ok.pdf

Valverde Berrocoso, J. (2011). Profesorado, tecnología educativa e innovación didáctica. En Valverde Berrocoso, J. (coord.) Docentes e-competentes. Buenas prácticas educativas con TIC (pp. 13-27). Barcelona: Octaedro. 


\section{(Endnotes)}

1 Este proyecto -desarrollado inicialmente en forma conjunta por el Ministerio de Educación y Cultura (MEC), la Administración Nacional de Telecomunicaciones (Antel) y el Laboratorio Tecnológico del Uruguay (LATU)- se inscribe a nivel nacional en el Programa de Equidad para el Acceso a la Información Digital (Peaid), según Decreto presidencial del 18 de abril de 2007. El mismo está inspirado en el trabajo desarrollado internacionalmente por la Organización One Laptop Per Child (OLPC) creada bajo el influjo del científico estadounidense Nicholas Negroponte.

${ }^{2}$ La escolaridad en el sistema educativo uruguayo comprende: Educación Inicial (dos años), Educación Primaria (seis años) y Educación Media, dividida a su vez en dos ciclos: Básico y Superior, de tres años de duración cada uno, que se desenvuelve en centros de Educación Secundaria y de Educación Técnico Profesional. 